\title{
On the Bethe states of the one-dimensional supersymmetric $t-J$ model with generic open boundaries
}

\author{
Pei Sun,,$^{a, b}$ Fakai Wen, ${ }^{a, b}$ Kun Hao, ${ }^{a, b}$ Junpeng Cao, ${ }^{c, d, e}$ Guang-Liang Li, ${ }^{f}$ \\ Tao Yang, ${ }^{a, b, 1}$ Wen-Li Yang ${ }^{a, b, g}$ and Kangjie Shi ${ }^{a, b}$ \\ ${ }^{a}$ Institute of Modern Physics, Northwest University, \\ 229 Taibai Beilu, Xian 710069, China \\ ${ }^{b}$ Shaanxi Key Laboratory for Theoretical Physics Frontiers, \\ 229 Taibai Beilu, Xian 710069, China \\ ${ }^{c}$ Beijing National Laboratory for Condensed Matter Physics, \\ Institute of Physics, Chinese Academy of Sciences, \\ 8 3rd South Street, Zhongguancun, Beijing 100190, China \\ ${ }^{d}$ School of Physical Sciences, University of Chinese Academy of Sciences, \\ Beijing, China \\ ${ }^{e}$ Collaborative Innovation Center of Quantum Matter, \\ Beijing, China \\ ${ }^{f}$ Department of Applied Physics, Xian Jiaotong University, \\ 28 Xianning West Road, Xian 710049, China \\ ${ }^{g}$ Beijing Center for Mathematics and Information Interdisciplinary Sciences, \\ Beijing, 100048, China \\ E-mail: sunpei@stumail.nwu.edu.cn, fakaiwen@126.com, haoke72@163.com, \\ junpengcao@iphy.ac.cn, leegl@mail.xjtu.edu.cn, yangt@nwu.edu.cn, \\ wlyang@nwu.edu.cn, kjshi@nwu.edu.cn
}

ABSTRACT: By combining the algebraic Bethe ansatz and the off-diagonal Bethe ansatz, we investigate the supersymmetric $t-J$ model with generic open boundaries. The eigenvalues of the transfer matrix are given in terms of an inhomogeneous $T-Q$ relation, and the corresponding eigenstates are expressed in terms of nested Bethe states which have welldefined homogeneous limit. This exact solution provides basis for further analyzing the thermodynamic properties and correlation functions of the model.

Keywords: Bethe Ansatz, Lattice Integrable Models

ARXIV EPRINT: 1705.09478

\footnotetext{
${ }^{1}$ Corresponding author.
} 


\section{Contents}

1 Introduction 1

2 Integrability of the model $\quad 2$

3 Nested algebraic Bethe ansatz 5

$\begin{array}{lll}4 & \text { Reduced spectrum problem } & 7\end{array}$

5 Nested inhomogeneous $T-Q$ relation $\quad 11$

6 Concluding remarks $\quad 12$

\section{Introduction}

The $t-J$ model is one of the cornerstones in the study of high- $T_{c}$ superconductivity [1], which is a large- $U$ limit of the single-band Hubbard model [2-5]. The Hamiltonian of the model have played essential roles in theoretical study of strongly correlated copperoxide based materials [6]. In general, the Hamiltonian of the supersymmetric $t-J$ model with the general boundary interaction terms is given by

$$
\begin{aligned}
H= & -t \sum_{\alpha, j=1}^{L-1} \mathcal{P}\left[c_{j, \alpha}^{+} c_{j+1, \alpha}+c_{j+1, \alpha}^{+} c_{j, \alpha}\right] \mathcal{P}+J \sum_{k=1}^{L-1}\left[\mathbf{S}_{k} \cdot \mathbf{S}_{k+1}-\frac{1}{4} n_{k} n_{k+1}\right]+\sum_{l=1}^{L-1} n_{l}+n_{l+1} \\
& -\mu \hat{N}+\xi_{1} n_{1}+2 h_{1}^{z} S_{1}^{z}+2 h_{1}^{-} S_{1}^{-}+2 h_{1}^{+} S_{1}^{+}+\xi_{L} n_{L}+2 h_{L}^{z} S_{L}^{z}+2 h_{L}^{-} S_{L}^{-}+2 h_{L}^{+} S_{L}^{+},
\end{aligned}
$$

where $t$ is the nearest neighbor hopping of electrons and $J$ is the antiferomagetic exchange; $L$ is the total number of lattice sites; the operators $c_{j, \sigma}$ and $c_{j, \sigma}^{+}$are the annihilation and creation operators of the electron with spin $\sigma= \pm 1$ on the lattice site $j$, which satisfies anticommutation relations, i.e., $\left\{c_{i, \sigma}^{+}, c_{j, \tau}\right\}=\delta_{i, j} \delta_{\sigma, \tau}$. There are only three possible states at the lattice site $i$ due to the factor $\mathcal{P}=\left(1-n_{j,-\sigma}\right)$ ruled out double occupancies; the operator $n_{j}=\sum_{\sigma= \pm} n_{j, \sigma}$ means the total number operator on site $j$ and $n_{j, \sigma}=c_{j, \sigma}^{+} c_{j, \sigma}$; $\mu$ is the chemical potential and $\hat{N}=\sum_{j=1}^{L} n_{j} ; \xi_{1, L}$ are the boundary chemical potentials; $h_{1, L}^{z}$ and $h_{1, L}^{ \pm}$are the boundary fields; the spin operators $S^{-}=\sum_{j=1}^{L} S_{j}^{-}, \quad S^{+}=\sum_{j=1}^{L} S_{j}^{+}$ and $S^{z}=\sum_{j=1}^{L} S_{j}^{z}$, form the $s u(2)$ algebra and can be expressed by

$$
S_{j}^{-}=c_{j, 1}^{+} c_{j,-1}, \quad S_{j}^{+}=c_{j,-1}^{+} c_{j}, \quad S_{j}^{z}=\frac{1}{2}\left(n_{j, 1}-n_{j,-1}\right) .
$$

It is well-known that the one-dimensional $t-J$ model is integrable at the supersymmetric point $J= \pm 2 t[7-9]$, and the model with the periodic boundary condition or the diagonal 
boundaries has been studied by employing many Bethe ansatz methods [10-20]. For the non-diagonal boundary case, the nested algebraic Bethe ansatz method doesn't work since the $\mathrm{U}(1)$ symmetry is broken. With the help of the off-diagonal Bethe ansatz [21-27], the exact energy spectrum of the one-dimensional supersymmetric $t-J$ model with unparallel boundary fields has been obtained [28]. However, the eigenstates (or Bethe states) which have played important roles in applications of the model are still missing.

In this paper, we study the supersymmetric $t-J$ model with generic integrable boundary conditions in grading: bosonic, fermionic and fermionic $(\mathrm{BFF})$. By combining the graded nested algebraic Bethe ansatz and off-diagonal Bethe ansatz, we obtain the Bethe states which have well-defined homogeneous limit and the corresponding eigenvalues of the transfer matrix of the model. Numerical results for the small size systems suggest that the spectrum obtained by the nested Bethe ansatz equations (BAEs) is complete.

The paper is organized as follows. In section 2, the associated graded $R$-matrix and corresponding generic integral non-diagonal boundary reflection matrices are introduced. In section 3, by using the graded algebraic Bethe ansatz, we derive the eigenvalues of the transfer matrix of the system which related with the eigenvalues of the nested transfer matrix. In section 4, the eigenvalues of the nested transfer matrix are derived by offdiagonal Bethe ansatz, and the Bethe states are also be given. In section 5 , we construct the nested inhomogeneous $T-Q$ relation and the nested Bethe ansatz equations of the supersymmetric $t-J$ model. Section 6 contains our results and give some discussions.

\section{$2 \quad$ Integrability of the model}

In this paper we consider $J=2 t=2$ which corresponds to the supersymmetric and integrable point [29]. The integrability of the model is associated with the rational $R$-matrix $R(u)$ given by

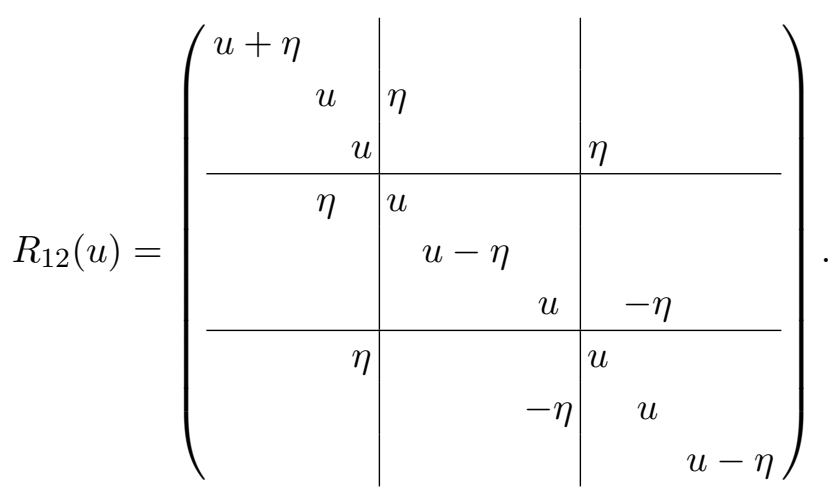

The $R$-matrix $R(u)$ possesses the following properties

$$
\begin{aligned}
& \text { Initial condition: } \quad R_{12}(0)=\eta P_{12}, \\
& \text { Unitarity relation: } \quad R_{12}(u) R_{21}(-u)=\rho_{1}(u) \times \mathrm{id} \text {, } \\
& \text { Crossing Unitarity relation: } \quad R_{12}^{s t_{1}}(-u+\eta) R_{21}^{s t_{1}}(u)=\rho_{2}(u) \times \text { id. }
\end{aligned}
$$

Here $P_{12}$ is the graded permutation operator with the definition

$$
P_{\beta_{1} \beta_{2}}^{\alpha_{1} \alpha_{2}}=(-1)^{p\left(\alpha_{1}\right) p\left(\alpha_{2}\right)} \delta_{\alpha_{1} \beta_{2}} \delta_{\beta_{1} \alpha_{2}},
$$


$p\left(\alpha_{i}\right)$ is the Grassmann parities which is one for fermions and zero for bosons. Here, we choose BFF grading which means $p(1)=0, p(2)=p(3)=1$ and $R_{21}(u)=P_{12} R_{12}(u) P_{12}$, $s t_{i}$ denotes the super transposition in the $i$-th space $\left(A^{s t}\right)_{i j}=A_{j i}(-1)^{p(i)[p(i)+p(j)]}$ and $i s t_{i}$ denotes the inverse super transposition. The functions $\rho_{1}(u)$ and $\rho_{2}(u)$ are given by

$$
\rho_{1}(u)=-(u-\eta)(u+\eta), \quad \rho_{2}(u)=-u(u-\eta) .
$$

Here and below we adopt the standard notations: for any matrix $A \in \operatorname{End}(\mathbf{V}), A_{j}$ is an super embedding operator in the $Z_{2}$ graded tensor space $\mathbf{V} \otimes \mathbf{V} \otimes \cdots$, which acts as $A$ on the $j$-th space and as identity on the other factor spaces. For $R \in \operatorname{End}(\mathbf{V} \otimes \mathbf{V}), R_{i j}$ is an super embedding operator of $R$ in the $Z_{2}$ graded tensor space, which acts as identity on the factor spaces except for the $i$-th and $j$-th ones. The super tensor product of two operators are defined through $(A \otimes B)_{\beta \delta}^{\alpha \gamma}=(-1)^{[p(\alpha)+p(\beta)] p(\gamma)} A_{\beta}^{\alpha} B_{\delta}^{\gamma}$. (For further details we refer the reader to [30]).

The $R$-matrix is an even operator (i.e., the parities of the non-zero matrix elements $R_{b d}^{a c}$ of the $R$-matrix satisfies $\left.p(a)+p(b)+p(c)+p(d)=0\right)$ and satisfies the graded quantum Yang-Baxter equation (QYBE)

$$
R_{12}(u-v) R_{13}(u) R_{23}(v)=R_{23}(v) R_{13}(u) R_{12}(u-v) .
$$

In terms of the matrix entries, it reads

$$
\begin{aligned}
& R(\lambda-u)_{\beta_{1} \beta_{2}}^{\alpha_{1} \alpha_{2}} R(\lambda)_{\gamma_{1} \beta_{3}}^{\beta_{1} \alpha_{3}} R(u)_{\gamma_{2} \gamma_{3}}^{\beta_{2} \beta_{3}}(-1)^{\left(p\left(\beta_{1}\right)+p\left(\gamma_{1}\right)\right) p\left(\beta_{2}\right)} \\
& \quad=R(u)_{\beta_{2} \beta_{3}}^{\alpha_{2} \alpha_{3}} R(\lambda)_{\beta_{1} \gamma_{3}}^{\alpha_{1} \beta_{3}} R(\lambda-u)_{\gamma_{1} \gamma_{2}}^{\beta_{1} \beta_{2}}(-1)^{\left(p\left(\alpha_{1}\right)+p\left(\beta_{1}\right)\right) p\left(\beta_{2}\right)} .
\end{aligned}
$$

Let us now introduce the reflection matrix $K^{-}(u)$ and its dual one $K^{+}(u)$. The former satisfies the graded reflection equation (RE) [31]

$$
\begin{aligned}
& R_{12}\left(u_{1}-u_{2}\right) K_{1}^{-}\left(u_{1}\right) R_{21}\left(u_{1}+u_{2}\right) K_{2}^{-}\left(u_{2}\right) \\
& \quad=K_{2}^{-}\left(u_{2}\right) R_{12}\left(u_{1}+u_{2}\right) K_{1}^{-}\left(u_{1}\right) R_{21}\left(u_{1}-u_{2}\right),
\end{aligned}
$$

and the latter satisfies the dual RE which take the form [32]

$$
\begin{aligned}
& R_{12}\left(u_{2}-u_{1}\right) K_{1}^{+}\left(u_{1}\right) \widetilde{\widetilde{R}}_{21}\left(-u_{1}-u_{2}\right)^{i s t_{1}, s t_{2}} K_{2}^{+}\left(u_{2}\right) \\
& \quad=K_{2}^{+}\left(u_{2}\right) \tilde{R}_{12}\left(-u_{1}-u_{2}\right)^{i s t_{1}, s t_{2}} K_{1}^{+}\left(u_{1}\right) R_{21}\left(u_{2}-u_{1}\right),
\end{aligned}
$$

where

$$
\begin{aligned}
& \widetilde{\widetilde{R}}_{21}(u)^{i s t_{1}, s t_{2}}=\left(\left[\left\{R_{21}^{-1}(u)\right\}^{i s t_{2}}\right]^{-1}\right)^{s t_{2}}, \\
& \tilde{R}_{12}(u)^{i s t_{1}, s t_{2}}=\left(\left[\left\{R_{12}^{-1}(u)\right\}^{s t_{1}}\right]^{-1}\right)^{i s t_{1}} .
\end{aligned}
$$

For our case, the dual reflection equation (2.10) reduces to

$$
\begin{aligned}
& R_{12}\left(u_{2}-u_{1}\right) K_{1}^{+}\left(u_{1}\right) R_{21}\left(-u_{1}-u_{2}+\eta\right) K_{2}^{+}\left(u_{2}\right) \\
& \quad=K_{2}^{+}\left(u_{2}\right) R_{12}\left(-u_{1}-u_{2}+\eta\right) K_{1}^{+}\left(u_{1}\right) R_{21}\left(u_{2}-u_{1}\right) .
\end{aligned}
$$


In this paper we consider the generic non-diagonal $K$-matrices $K^{-}(u)$

$$
K^{-}(u)=\left(\begin{array}{ccc}
\zeta+(2 c-1) u & 0 & 0 \\
0 & \zeta-u & 2 c_{1} u \\
0 & 2 c_{2} u & \zeta+u
\end{array}\right) \equiv\left(\begin{array}{ccc}
k_{11}^{-} & 0 & 0 \\
0 & k_{22}^{-} & k_{23}^{-} \\
0 & k_{32}^{-} & k_{33}^{-}
\end{array}\right) .
$$

Here the four boundary parameters $c, c_{1}, c_{2}$ and $\zeta$ are not independent with each other, and satisfy a constraint

$$
c^{2}=c_{1} c_{2}+c .
$$

The dual non-diagonal reflection matrix $K^{+}(u)$ is given by

$$
K^{+}(u)=\left.K^{-}(-u+\eta / 2)\right|_{\left(\zeta, c, c_{1}, c_{2}\right) \rightarrow\left(\zeta^{\prime}, c^{\prime}, c_{1}^{\prime}, c_{2}^{\prime}\right)} \equiv\left(\begin{array}{ccc}
k_{11}^{+} & 0 & 0 \\
0 & k_{22}^{+} & k_{23}^{+} \\
0 & k_{32}^{+} & k_{33}^{+}
\end{array}\right),
$$

with the constraint

$$
c^{\prime 2}=c_{1}^{\prime} c_{2}^{\prime}+c^{\prime}
$$

In order to show the integrability of the system, we first introduce the "row-to-row" monodromy matrices $T_{0}(u)$ and $\hat{T}_{0}(u)$

$$
\begin{aligned}
& T_{0}(u)=R_{0 L}\left(u-\theta_{L}\right) R_{0 L-1}\left(u-\theta_{L-1}\right) \cdots R_{01}\left(u-\theta_{1}\right), \\
& \hat{T}_{0}(u)=R_{10}\left(u+\theta_{1}\right) R_{20}\left(u+\theta_{2}\right) \cdots R_{L 0}\left(u+\theta_{L}\right),
\end{aligned}
$$

where $\left\{\theta_{j}, j=1 \cdots L\right\}$ are the inhomogeneous parameters and $L$ is the number of sites. The one-row monodromy matrices are the $3 \times 3$ matrices in the auxillary space 0 and their elements act on the quantum space $\mathbf{V}^{\otimes L}$. The tensor product is in the graded space, so we can write

$$
\begin{aligned}
\left\{[T(u)]^{a b}\right\}_{\beta_{1} \ldots \beta_{L}}^{\alpha_{1} \ldots \alpha_{L}}= & R_{0 N}(u)_{c_{L} \beta_{L}}^{a \alpha_{L}} \ldots R_{0 j}(u)_{c_{j} \beta_{j}}^{c_{j+1} \alpha_{j}} \ldots R_{01}(u)_{b \beta_{1}}^{c_{2} \alpha_{1}} \\
& \times(-1)^{\sum_{j=2}^{L}\left(p\left(\alpha_{j}\right)+p\left(\beta_{j}\right)\right) \sum_{i=1}^{j-1} p\left(\alpha_{i}\right)} .
\end{aligned}
$$

For the system with open boundaries, we need to define the double-row monodromy matrix

$$
\mathbb{T}_{0}(u)=T_{0}(u) K_{0}^{-}(u) \hat{T}_{0}(u),
$$

which satisfies the similar relation as (2.9), in terms of matrix entries, they are

$$
\begin{aligned}
& R(u-\lambda)_{b_{1} b_{2}}^{a_{1} a_{2}} \mathbb{T}(u)_{c_{1}}^{b_{1}} R(u+\lambda)_{c_{2} d_{1}}^{b_{2} c_{1}} \mathbb{T}(\lambda)_{d_{2}}^{c_{2}}(-1)^{\left(p\left(b_{1}\right)+p\left(c_{1}\right)\right) p\left(b_{2}\right)} \\
& \quad=\mathbb{T}(\lambda)_{b_{2}}^{a_{2}} R(u+\lambda)_{b_{1} c_{2}}^{a_{1} b_{2}} \mathbb{T}(u)_{c_{1}}^{b_{1}} R(u-\lambda)_{d_{2} d_{1}}^{c_{2} c_{1}}(-1)^{\left(p\left(b_{1}\right)+p\left(c_{1}\right)\right) p\left(c_{2}\right)} .
\end{aligned}
$$

Then the transfer matrix of the system is constructed as

$$
t(u)=\operatorname{str}_{0}\left\{K_{0}^{+}(u) \mathbb{T}_{0}(u)\right\}=\sum_{\alpha=1}^{3}(-1)^{p(\alpha)}\left[K_{0}^{+}(u) \mathbb{T}_{0}(u)\right]_{\alpha \alpha} .
$$


By using the (2.8), (2.9) and (2.10), we can prove the commutativity of $t(u)$. (For further details about the commuting transfer matrix with boundaries for graded case, we refer the reader to $[14,32,33])$. The Hamiltonian (1.1) can be constructed by taking the derivative of the logarithm of the transfer matrix $t(u)$ of the system

$$
H=-\left.\frac{\eta}{2} \frac{\partial \ln t(u)}{\partial u}\right|_{u=0,\left\{\theta_{j}=0\right\}}+\frac{\eta(2 c-1)}{2 \zeta}+\frac{\zeta^{\prime}}{\left(c^{\prime}-1 / 2\right) \eta-\zeta^{\prime}}-\mu \hat{N}+L-1,
$$

with the parameters chosen as follows:

$$
\begin{aligned}
& \xi_{1}=-\frac{\eta}{2 \zeta}(1-2 c), \quad h_{1}^{z}=-\frac{\eta}{2 \zeta}, \quad h_{1}^{-}=-\frac{\eta}{2 \zeta} c_{2}, \quad h_{1}^{+}=-\frac{\eta}{2 \zeta} c_{1}, \quad \xi_{L}=\frac{\left(c^{\prime}-1 / 2\right) \eta}{\left(c^{\prime}-1 / 2\right) \eta-\zeta^{\prime}}, \\
& h_{L}^{z}=\frac{-\eta / 2}{\left(c^{\prime}-1 / 2\right) \eta-\zeta^{\prime}}, \quad h_{L}^{-}=\frac{-\eta c_{2}^{\prime} / 2}{\left(c^{\prime}-1 / 2\right) \eta-\zeta^{\prime}} \quad \text { and } \quad h_{L}^{+}=\frac{-\eta c_{1}^{\prime} / 2}{\left(c^{\prime}-1 / 2\right) \eta-\zeta^{\prime}} .
\end{aligned}
$$

\section{$3 \quad$ Nested algebraic Bethe ansatz}

The block-diagonal structure of the $K$-matrix (2.14) permits us to use the nested algebraic Bethe ansatz to construct the associated Bethe state and obtain the eigenvalue as follows. We first represent the double-row monodromy matrix $\mathbb{T}_{0}(u)=T_{0}(u) K_{0}^{-}(u) \hat{T}_{0}(u)$ in the form

$$
\mathbb{T}_{0}(u)=\left(\begin{array}{ccc}
A(u) & B_{1}(u) & B_{2}(u) \\
C_{1}(u) & D_{11}(u) & D_{12}(u) \\
C_{2}(u) & D_{21}(u) & D_{22}(u)
\end{array}\right) .
$$

Then the transfer matrix can be expressed by

$$
t(u)=\left[k_{11}^{+}(u) A(u)-\sum_{i, j=1}^{2} k_{i+1, j+1}^{+}(u) D_{j i}(u)\right],
$$

where $k_{i j}^{ \pm}$is the $K^{ \pm}$matrix element in the $i$ th row and $j$ th column.

Now we use the graded version of the nested algebraic Bethe ansatz method to obtain the eigenvalues of the transfer matrix (3.2). For this purpose, we first define the reference state $\left|\Psi_{0}\right\rangle$ as

$$
\left|\Psi_{0}\right\rangle=\bigotimes_{j=1}^{L}|0\rangle_{j}, \quad|0\rangle_{j}=\left(\begin{array}{l}
1 \\
0 \\
0
\end{array}\right) .
$$

From the relations $(2.21),(3.1)$ and (3.3), the elements of matrix $\mathbb{T}_{0}(u)$ acting on the reference state $\left|\Psi_{0}\right\rangle$ give rise to

$$
\begin{aligned}
A(u)\left|\Psi_{0}\right\rangle & =k_{11}^{-}(u) a_{0}(u)\left|\Psi_{0}\right\rangle, \\
D_{11}(u)\left|\Psi_{0}\right\rangle & =\left\{\frac{\eta}{2 u+\eta} k_{11}^{-}(u) a_{0}(u)+\left[k_{22}^{-}(u)-\frac{\eta}{2 u+\eta} k_{11}^{-}(u)\right] b_{0}(u)\right\}\left|\Psi_{0}\right\rangle, \\
D_{22}(u)\left|\Psi_{0}\right\rangle & =\left\{\frac{\eta}{2 u+\eta} k_{11}^{-}(u) a_{0}(u)+\left[k_{33}^{-}(u)-\frac{\eta}{2 u+\eta} k_{11}^{-}(u)\right] b_{0}(u)\right\}\left|\Psi_{0}\right\rangle, \\
D_{12}(u)\left|\Psi_{0}\right\rangle & =k_{23}^{-}(u) b_{0}(u)\left|\Psi_{0}\right\rangle \\
D_{21}(u)\left|\Psi_{0}\right\rangle & =k_{32}^{-}(u) b_{0}(u)\left|\Psi_{0}\right\rangle, \\
B_{i}(u)\left|\Psi_{0}\right\rangle & \neq 0, \quad C_{i}(u)\left|\Psi_{0}\right\rangle=0, \quad i=1,2
\end{aligned}
$$


where

$$
b_{0}(u)=\prod_{j=1}^{L}\left(u-\theta_{j}\right)\left(u+\theta_{j}\right), \quad a_{0}(u)=b_{0}(u+\eta) .
$$

The operators $B_{1}(u)$ and $B_{2}(u)$ acting on the reference state give nonzero values, and can be regarded as the creation operators of the eigenstates of the system. Following the procedure of the nested algebraic Bethe ansatz, the eigenstates of the transfer matrix can be constructed as

$$
\left|u_{1}, \ldots, u_{M} ; \mathcal{F}\right\rangle=B_{a_{1}}\left(u_{1}\right) B_{a_{2}}\left(u_{2}\right) \ldots B_{a_{M}}\left(u_{M}\right) \mathcal{F}^{a_{1} a_{2} \ldots a_{M}}\left|\Psi_{0}\right\rangle
$$

where we have used the convention that the repeated indices indict the sum over the values 1,2 , and $\mathcal{F}^{a_{1} \ldots a_{n}}$ is a function of the spectral parameters $u_{j}$. Moreover, the coefficients $\mathcal{F}^{a_{1} \ldots a_{n}}$ are actually the vector components of the nested Bethe state (see below (4.27)). As the transfer matrix (3.2) acting on the assumed states (3.6), we should exchange the positions of the operators $A(u), D_{i j}(u)$ and the operators $B_{a_{j}}\left(u_{j}\right)$. With the help of the reflection equation (2.20) and the Yang-Baxter equation (2.8), we can derive commutation relations

$$
\begin{aligned}
B_{i}(u) B_{j}(v)= & -B_{k}(v) B_{l}(u) \frac{r_{l k}^{i j}(u-v)}{u-v+\eta} \\
A(u) B_{j}(v)= & \frac{(u-v-\eta)(u+v)}{(u+v+\eta)(u-v)} B_{j}(v) A(u)-\frac{\eta}{u+v+\eta} B_{i}(u) \tilde{D}_{i j}(v) \\
& +\frac{2 v \eta}{(u-v)(2 v+\eta)} B_{j}(u) A(v), \\
\tilde{D}_{i j}(u) B_{k}(v)= & \frac{r_{e f}^{i d}(u+v+\eta) r_{k j}^{f g}(u-v)}{(u+v+\eta)(u-v)} B_{d}(v) \tilde{D}_{e g}(u)+\eta \frac{r_{e j}^{i d}(2 u+\eta)}{(2 u+\eta)(u-v)} B_{d}(u) \tilde{D}_{e k}(v) \\
& -\frac{2 v \eta}{2 u+\eta} \frac{r_{k j}^{i d}(2 u+\eta)}{(2 v+\eta)(u+v+\eta)} B_{d}(u) A(v),
\end{aligned}
$$

where $r_{i j}=u+\eta \mathbb{P}_{i j}, \mathbb{P}_{\beta_{1} \beta_{2}}^{\alpha_{1} \alpha_{2}}=(-1)^{p\left(\alpha_{1}\right) p\left(\beta_{2}\right)} \delta_{\alpha_{1} \beta_{2}} \delta_{\beta_{1} \alpha_{2}}$ with the grading $p^{(1)}=p^{(2)}=1$, and

$$
\tilde{D}_{i j}(u)=D_{i j}(u)-\delta_{i j} \frac{\eta}{2 u+\eta} A(u) .
$$

Acting the transfer matrix $t(u)$ on the state $|\Psi\rangle$ and repeatedly using the commutation relations (3.8) and (3.9), we obtain

$$
t(u)\left|u_{1}, \ldots, u_{M} ; \mathcal{F}\right\rangle=\Lambda(u)\left|u_{1}, \ldots, u_{M} ; \mathcal{F}\right\rangle+\text { unwanted terms }
$$

where the corresponding eigenvalue $\Lambda(u)$ is

$$
\begin{aligned}
\Lambda(u)= & {\left[-\frac{\eta}{2 u+\eta} \sum_{i=2}^{3} k_{i i}^{+}(u)+k_{11}^{+}(u)\right] k_{11}^{-}(u) a_{0}(u) \prod_{i=1}^{M} \frac{\left(u-u_{i}-\eta\right)}{\left(u-u_{i}\right)} \frac{\left(u+u_{i}\right)}{\left(u+u_{i}+\eta\right)} } \\
& -b_{0}(u) \prod_{i=1}^{M} \frac{1}{\left(u-u_{i}\right)\left(u+u_{i}+\eta\right)} \hat{\Lambda}\left(u,\left\{u_{j}\right\}\right),
\end{aligned}
$$


and $\hat{\Lambda}\left(u,\left\{u_{j}\right\}\right)$ is the eigenvalue of the nested transfer matrix $\hat{t}\left(u,\left\{u_{j}\right\}\right)$ given by

$$
\begin{aligned}
\hat{t}\left(u,\left\{u_{j}\right\}\right)= & \frac{2 u}{2 u+\eta} \operatorname{tr}_{\overline{0}}\left[\bar{K}_{\overline{0}}^{+}(u) r_{\overline{0} 1}\left(u+u_{1}+\eta\right) \ldots r_{\overline{0} M}\left(u+u_{M}+\eta\right)\right. \\
& \left.\times \bar{K}_{\overline{0}}^{-}(u) r_{M \overline{0}}\left(u-u_{M}\right) \ldots r_{1 \overline{0}}\left(u-u_{1}\right)\right], \\
\bar{K}^{+}(u)= & \left(\begin{array}{cc}
k_{22}^{+}(u) & k_{23}^{+}(u) \\
k_{32}^{+}(u) & k_{33}^{+}(u)
\end{array}\right), \\
\bar{K}^{-}(u)= & \frac{2 u+\eta}{2 u}\left(\begin{array}{cc}
k_{22}^{-}(u)-\frac{\eta}{2 u+\eta} k_{11}^{-}(u) & k_{23}^{-}(u) \\
k_{32}^{-}(u) & k_{33}^{-}(u)-\frac{\eta}{2 u+\eta} k_{11}^{-}(u)
\end{array}\right),
\end{aligned}
$$

namely,

$$
\hat{t}\left(u,\left\{u_{j}\right\}\right)|\mathcal{F}\rangle=\hat{\Lambda}\left(u,\left\{u_{j}\right\}\right)|\mathcal{F}\rangle .
$$

The vector components $\left\{F^{a_{1} \ldots a_{n}}\right\}$ allow us to reconstruct the associated Bethe state (3.6), while the eigenvalue $\hat{\Lambda}\left(u,\left\{u_{j}\right\}\right)$ gives rise to the associated eigenvalue (3.12) of the transfer matrix $t(u)$ of the model. We shall determine the eigenvalue $\hat{\Lambda}\left(u,\left\{u_{j}\right\}\right)$ and the corresponding eigenstate $|\mathcal{F}\rangle$ in the next section. The condition that the unwanted terms should be zero gives rise to that the $M$ Bethe roots must satisfy the associated Bethe ansatz equations (BAEs)

$$
1=\frac{K^{(1)}\left(u_{k}\right) a_{0}\left(u_{k}\right) Q^{(1)}\left(u_{k}-\eta\right)}{\left(2 u_{k}+\eta\right) b_{0}\left(u_{k}\right) \hat{\Lambda}\left(u_{k},\left\{u_{j}\right\}\right)}, \quad k=1 \ldots M
$$

where

$$
\begin{aligned}
& Q^{(1)}(u)=\prod_{i=1}^{M}\left(u-u_{i}\right)\left(u+u_{i}+\eta\right) \\
& K^{(1)}(u)=\left(\left(2-4 c^{\prime}\right) u^{2}+2 \zeta^{\prime} u-\eta \zeta^{\prime}-\frac{1}{2} \eta^{2}+\eta^{2} c^{\prime}\right)(\zeta+(2 c-1) u) .
\end{aligned}
$$

Some remarks are in order. It is easy to check that the nested Bethe state $\left|u_{1}, \ldots, u_{M} ; \mathcal{F}\right\rangle$ given by (3.6) and the eigenvalue $\Lambda(u)$ given by (3.12) both have well-defined homogeneous limit (i.e., $\theta_{j} \rightarrow 0$ ). This implies that in the homogeneous limit, the resulting Bethe states and the eigenvalue give rise to the eigenstate and the corresponding eigenvalue of the super $t-J$ model described by the Hamiltonian (1.1).

\section{Reduced spectrum problem}

In the previous section, we have reduced searching eigenstates of the original transfer matrix $t(u)(2.21)$ into the spectrum problem (3.16) of the nested transfer matrix $\hat{t}\left(u,\left\{u_{j}\right\}\right)$ given by (3.13). Now, we are in the position to calculate the eigenvalue $\hat{\Lambda}\left(u,\left\{u_{j}\right\}\right)$ and the corresponding eigenstate $|\mathcal{F}\rangle$ of the nested transfer matrix $\hat{t}\left(u,\left\{u_{j}\right\}\right)$ which allows us to reconstruct the Bethe state (3.6) of the supersymmetric $t-J$ model. Because the reflection matrices (3.14) and (3.15) have the off-diagonal elements. The traditional algebraic Bethe ansatz is invalid [22] due to the fact that the system doesnot have the obvious reference 
state. Thanks to the works [34-37], we can solve the spectrum problem (3.16) as follows. For simplicity, let $\lambda=u+\frac{1}{2} \eta$ and $\lambda_{j}=u_{j}+\frac{1}{2} \eta$. We recognize the $\hat{t}\left(u,\left\{u_{j}\right\}\right)$ as the transfer matrix of the open spin- $1 / 2$ XXX chain of length $M$ with non-diagonal boundary terms. Following the procedure in [34]

$$
\begin{aligned}
\hat{t}\left(\lambda,\left\{\lambda_{j}\right\}\right) & =\frac{2 \lambda-\eta}{2 \lambda} \bar{t}\left(\lambda,\left\{\lambda_{j}\right\}\right) \\
& =\frac{2 \lambda-\eta}{2 \lambda} \operatorname{Tr}_{\overline{0}}\left\{\bar{K}_{\overline{0}}^{+}(\lambda) \bar{T}_{\overline{0}}\left(\lambda,\left\{\lambda_{j}\right\}\right) \bar{K}_{\overline{0}}^{-}(\lambda) \widehat{\bar{T}}_{\overline{0}}\left(\lambda,\left\{\lambda_{j}\right\}\right)\right\},
\end{aligned}
$$

where

$$
\begin{aligned}
\bar{T}_{\overline{0}}\left(\lambda,\left\{\lambda_{j}\right\}\right) & =r_{\overline{0} 1}\left(\lambda+\lambda_{1}\right) \ldots r_{\overline{0} M}\left(\lambda+\lambda_{M}\right), \\
\widehat{\bar{T}}_{\overline{0}}\left(\lambda,\left\{\lambda_{j}\right\}\right) & =r_{M \overline{0}}\left(\lambda-\lambda_{M}\right) \ldots r_{1 \overline{0}}\left(\lambda-\lambda_{1}\right), \\
\bar{K}^{+}(\lambda) & =\left(\begin{array}{cc}
\zeta^{\prime}+\lambda-\eta & 2 c_{1}^{\prime}(-\lambda+\eta) \\
2 c_{2}^{\prime}(-\lambda+\eta) & \zeta^{\prime}-\lambda+\eta
\end{array}\right), \\
\bar{K}^{-}(\lambda) & =\left(\begin{array}{cr}
-\lambda+1 / 2 \eta+\zeta-c \eta & 2 c_{1} \lambda \\
2 c_{2} \lambda & \lambda+1 / 2 \eta+\zeta-c \eta
\end{array}\right) .
\end{aligned}
$$

We have checked that (4.4) is the solution of the normal RE of the following form

$$
\begin{aligned}
& r_{12}\left(u_{1}-u_{2}\right) \bar{K}_{1}^{-}\left(u_{1}\right) r_{21}\left(u_{1}+u_{2}\right) \bar{K}_{2}^{-}\left(u_{2}\right) \\
& \quad=\bar{K}_{2}^{-}\left(u_{2}\right) r_{12}\left(u_{1}+u_{2}\right) \bar{K}_{1}^{-}\left(u_{1}\right) r_{21}\left(u_{1}-u_{2}\right),
\end{aligned}
$$

and that (4.3) satisfies the dual one. The $r$-matrix possesses the properties

$$
\begin{aligned}
\text { Initial condition: } & r_{12}(0)=-\eta \bar{P}_{12}, \\
\text { Unitary relation: } & r_{12}(\lambda) r_{21}(-\lambda)=\bar{\rho}_{1}(\lambda) \times \mathrm{id}, \\
\text { Crossing Unitary relation: } & r_{12}^{t_{1}}(\lambda) r_{21}^{t_{1}}(-\lambda+2 \eta)=\bar{\rho}_{2}(\lambda) \times \mathrm{id}, \\
\text { PT-symmetry: } & r_{21}(\lambda)=r_{12}^{t_{1} t_{2}}(\lambda) .
\end{aligned}
$$

Here $r_{21}(\lambda)=\bar{P}_{12} r_{12}(\lambda) \bar{P}_{12}$ and $\bar{P}_{\beta_{1} \beta_{2}}^{\alpha_{1} \alpha_{2}}=\delta_{\alpha_{1} \beta_{2}} \delta_{\beta_{1} \alpha_{2}}$. The functions $\overline{\rho_{1}}(\lambda)$ and $\overline{\rho_{2}}(\lambda)$ are given by

$$
\overline{\rho_{1}}(\lambda)=-(\lambda-\eta)(\lambda+\eta), \quad \overline{\rho_{2}}(\lambda)=-\lambda(\lambda-2 \eta) .
$$

From the definition (4.1), we know that the eigenvalue $\bar{\Lambda}(\lambda)$ of the transfer matrix $\bar{t}\left(\lambda,\left\{\lambda_{j}\right\}\right)$ is a polynomial of $\lambda$ and satisfies the relations:

Crossing symmetry : $\quad \bar{\Lambda}(\lambda)=\bar{\Lambda}(-\lambda+\eta)$,

Asymptotic behavior : $\bar{\Lambda}(\lambda) \sim\left(-2-4 c_{1} c_{2}^{\prime}-4 c_{1}^{\prime} c_{2}\right) u^{2 M+2}, \quad \lambda \rightarrow \infty$,

and

$$
\bar{\Lambda}\left(\lambda_{j}\right) \bar{\Lambda}\left(\lambda_{j}+\eta\right)=\frac{\triangle_{q}\left(\lambda_{j}\right)}{\left(\eta-2 \lambda_{j}\right)\left(\eta+2 \lambda_{j}\right)}, \quad j=1, \cdots, M
$$


where

$$
\begin{aligned}
\Delta_{q}(\lambda)= & (2 \eta+2 \lambda)(2 \eta-2 \lambda) \\
& \times\left(\xi^{\prime 2}-\left(1+4 c_{1}^{\prime} c_{2}^{\prime}\right) \lambda^{2}\right)\left(\left(\xi+\frac{1}{2} \eta-c \eta\right)^{2}-\left(1+4 c_{1} c_{2}\right) \lambda^{2}\right) \\
& \times \prod_{j=1}^{M}\left(\lambda+\lambda_{j}-\eta\right)\left(\lambda-\lambda_{j}-\eta\right)\left(\lambda-\lambda_{j}+\eta\right)\left(\lambda+\lambda_{j}+\eta\right) .
\end{aligned}
$$

Some special points can also be calculated directly by using the properties of the $r$-matrix and the reflection matrices $\bar{K}^{( \pm)}(u)$ as:

$$
\begin{aligned}
& \bar{\Lambda}(0)=\prod_{l=1}^{M} \overline{\rho_{1}}\left(\lambda_{l}\right) \operatorname{tr}\left\{\bar{K}^{+}(0)\right\} \bar{K}^{-}(0) \times \mathrm{id}, \\
& \bar{\Lambda}(\eta)=\prod_{l=1}^{M} \bar{\rho}_{2}\left(\lambda_{l}+\eta\right) \operatorname{tr}\left\{\bar{K}^{-}(\eta)\right\} \bar{K}^{+}(\eta) \times \mathrm{id} .
\end{aligned}
$$

It is remarked that the above relations were derived independently by the Separation of Variables [38]. These conditions (4.11)-(4.16) allow us to construct the eigenvalue $\hat{\Lambda}(\lambda)$ in terms of an inhomogeneous $T-Q$ relation as $[34,35]$

$$
\begin{aligned}
\hat{\Lambda}(\lambda)= & \frac{(2 \lambda-\eta)}{2 \lambda} \bar{\Lambda}(\lambda) \\
= & \frac{(2 \lambda-2 \eta)}{2 \lambda} K^{(2)}(\lambda) \bar{a}(\lambda) \frac{Q^{(2)}(\lambda+\eta)}{Q^{(2)}(\lambda)}+K^{(3)}(\lambda) \bar{d}(\lambda) \frac{Q^{(2)}(\lambda-\eta)}{Q^{(2)}(\lambda)} \\
& +(2 \lambda-\eta)(2 \lambda-2 \eta) \bar{a}(\lambda) \bar{a}(-\lambda+\eta) \frac{h}{Q^{(2)}(\lambda)},
\end{aligned}
$$

where

$$
\begin{aligned}
\bar{a}(\lambda)= & \prod_{j=1}^{M}\left(\lambda+\lambda_{j}-\eta\right)\left(\lambda-\lambda_{j}-\eta\right), \\
\bar{d}(\lambda)= & \prod_{j=1}^{M}\left(\lambda-\lambda_{j}\right)\left(\lambda+\lambda_{j}\right), \\
K^{(2)}(\lambda)= & \left(-\sqrt{1+4\left(c^{\prime 2}-c^{\prime}\right)} \lambda+\zeta^{\prime}\right) \\
& \times\left(\sqrt{1+4\left(c^{2}-c\right)} \lambda+\zeta+1 / 2 \eta-c \eta\right), \\
K^{(3)}(\lambda)= & \left(\sqrt{1+4\left(c^{\prime 2}-c^{\prime}\right)}(\lambda-\eta)+\zeta^{\prime}\right) \\
& \times\left(\sqrt{1+4\left(c^{2}-c\right)}(-\lambda+\eta)+\zeta+1 / 2 \eta-c \eta\right), \\
h= & \frac{1}{2}\left(-1-2\left(c_{1}^{\prime} c_{2}+c_{2}^{\prime} c_{1}\right)+\sqrt{\left(1+4 c_{1}^{\prime} c_{2}^{\prime}\right)\left(1+4 c_{1} c_{2}\right)}\right), \\
Q^{(2)}(\lambda)= & \prod_{j=1}^{M}\left(\lambda-w_{j}\right)\left(\lambda+w_{j}-\eta\right) \stackrel{\text { def }}{=} \prod_{j=1}^{M}\left(\lambda-\nu_{j}-\frac{1}{2} \eta\right)\left(\lambda+\nu_{j}-\frac{1}{2} \eta\right) .
\end{aligned}
$$


Such parametrization obviously satisfies the crossing symmetry (4.11), asymptotic behavior (4.12), production identity (4.13) and the values of the special points (4.15) and (4.16). To ensure $\hat{\Lambda}(\lambda)$ to be a polynomial, the residues of $\hat{\Lambda}(\lambda)$ at the poles $w_{j}$ must vanish, i.e., the $M$ Bethe roots must satisfy the BAEs

$$
\begin{aligned}
& \left(2 w_{j}-2 \eta\right) K^{(2)}\left(w_{j}\right) \bar{a}\left(w_{j}\right) Q^{(2)}\left(w_{j}+\eta\right)+2 w_{j} K^{(3)}\left(w_{j}\right) \bar{d}\left(w_{j}\right) Q^{(2)}\left(w_{j}-\eta\right) \\
& +2 w_{j}\left(2 w_{j}-\eta\right)\left(2 w_{j}-2 \eta\right) \bar{a}\left(w_{j}\right) \bar{a}\left(-w_{j}+\eta\right) h=0 .
\end{aligned}
$$

Now, we construct the eigenstates $|\mathcal{F}\rangle$ of the nested transfer matrix $\hat{t}(\lambda)$. Following the ideas in [36, 37], we first introduce two transformation matrices $g^{( \pm)}$

$$
g^{(-)}=\left(\begin{array}{cc}
-1 & \frac{2 c_{1}}{1-\sqrt{1+4 c_{1} c_{2}}} \\
1 & \frac{-2 c_{1}}{1+\sqrt{1+4 c_{1} c_{2}}}
\end{array}\right), \quad g^{(+)}=\left(\begin{array}{cc}
-m & \sqrt{1+m n}-1 \\
-m & -\sqrt{1+m n}-1
\end{array}\right),
$$

where $m=\frac{-4 c_{1} c_{2}-\left(2 c_{1} c_{2}^{\prime}-2 c_{1}^{\prime} c_{2}\right) \sqrt{1+4 c_{1} c_{2}}+2 c_{1} c_{2}^{\prime}+2 c_{1}^{\prime} c_{2}}{\left(1+2 c_{1} c_{2}^{\prime}+2 c_{1}^{\prime} c_{2}\right)\left(\sqrt{1+4 c_{1} c_{2}}+1\right)}$ and $n=\frac{-4 c_{1} c_{2}-\left(2 c_{1}^{\prime} c_{2}-2 c_{1} c_{2}^{\prime}\right) \sqrt{1+4 c_{1} c_{2}}+2 c_{1}^{\prime} c_{2}+2 c_{1} c_{2}^{\prime}}{\left(1+2 c_{1} c_{2}^{\prime}+2 c_{1}^{\prime} c_{2}\right)\left(\sqrt{1+4 c_{1} c_{2}}-1\right)}$. The gauge matrices diagonalize the nested $K$-matrix $\bar{K}^{-}(\lambda)$ given by (4.3) and the matrix $g^{(-)} \bar{K}^{+}(\lambda)\left\{g^{(-)}\right\}^{-1}$ respectively, namely,

$$
\begin{aligned}
& g^{(+)}\left\{g^{(-)} \bar{K}^{+}(\lambda)\left\{g^{(-)}\right\}^{-1}\right\}\left\{g^{(+)}\right\}^{-1} \\
& =-\frac{1+2 c_{1} c_{2}^{\prime}+2 c_{1}^{\prime} c_{2}}{\sqrt{1+4 c_{1} c_{2}}}\left(\begin{array}{cc}
\sqrt{1+m n}(\lambda-\eta)-\frac{\sqrt{1+4 c_{1} c_{2}}}{1+2 c_{1} c_{2}^{\prime}+2 c_{1}^{\prime} c_{2}} \xi^{\prime} & 0 \\
0 & -\sqrt{1+m n}(\lambda-\eta)-\frac{\sqrt{1+4 c_{1} c_{2}}}{1+2 c_{1} c_{2}^{\prime}+2 c_{1}^{\prime} c_{2}} \xi^{\prime}
\end{array}\right) \text {, } \\
& g^{(-)} \bar{K}^{-}(\lambda)\left\{g^{(-)}\right\}^{-1}=\left(\begin{array}{cc}
1 / 2 \eta+\xi-c \eta+\lambda \sqrt{1+4 c_{1} c_{2}} & 0 \\
0 & 1 / 2 \eta+\xi-c \eta-\lambda \sqrt{1+4 c_{1} c_{2}}
\end{array}\right) .
\end{aligned}
$$

With the gauge transformation, we can introduce the gauged monodromy matrix $\mathbb{U}(\lambda)$

$$
\mathbb{U}(\lambda)=g^{(+)} \bar{T}(\lambda)\left\{g^{(-)} \bar{K}^{-}(\lambda)\left\{g^{(-)}\right\}^{-1}\right\} \hat{\bar{T}}(\lambda)\left\{g^{(+)}\right\}^{-1}=\left(\begin{array}{cc}
\mathbb{A}(\lambda) & \mathbb{B}(\lambda) \\
\mathbb{C}(\lambda) & \mathbb{D}(\lambda)
\end{array}\right) .
$$

Then it was shown in $[36,37]$ that the eigenstate $|\mathcal{F}\rangle$ in $(3.16)$ can be expressed as

$$
|\mathcal{F}\rangle=\bigotimes_{j=1}^{M}\left\{g_{(j)}^{(-)}\right\}^{-1} \prod_{j=1}^{M} \mathbb{B}\left(w_{j}\right)|0\rangle=\sum_{a_{i}=1,2} \mathcal{F}^{a_{1} a_{2} \ldots a_{M}}\left|a_{1}, \ldots, a_{M}\right\rangle,
$$

where the reference state $|0\rangle$ is

$$
|0\rangle=\bigotimes_{j=1}^{M}|1\rangle_{j}, \quad|1\rangle_{j}=\left(\begin{array}{l}
1 \\
0
\end{array}\right),
$$

provided that the parameters $\left\{w_{j} \mid j=1, \ldots, M\right\}$ satisfy the BAEs (4.24). The corresponding vector components $\left\{\mathcal{F}^{a_{1} a_{2} \ldots a_{M}}\right\}$ allow us to reconstruct the eigenstates $\left|u_{1}, \ldots, u_{M} ; \mathcal{F}\right\rangle$ given by (3.6) of the original system. ${ }^{1}$

\footnotetext{
${ }^{1}$ We have numerically checked, for small-site cases (such as $L=2,3$ ), that the states constructed by (3.6) with vector components $\left\{\mathcal{F}^{a_{1} a_{2} \ldots a_{M}}\right\}$ given by (4.27) give rise to the complete set of eigenstates of the transfer matrix $t(u)$ given by (2.21), provided that the parameters $\left\{u_{j}\right\}$ and $\left\{v_{j}\right\}$ (or $\left\{w_{j}\right\}$ ) satisfy the BAEs (5.2)-(5.3).
} 


\section{$5 \quad$ Nested inhomogeneous $T-Q$ relation}

Now we are ready to write out the eigenvalues $\Lambda(u)$ of the transfer matrices $t(u)$ in terms of some inhomogeneous $T-Q$ relation with the help of (3.12) and (4.17) as ${ }^{2}$

$$
\begin{aligned}
\Lambda(u)= & \frac{1}{(2 u+\eta)} K^{(1)}(u) a_{0}(u) \frac{Q^{(1)}(u-\eta)}{Q^{(1)}(u)} \\
& -\frac{(2 u-\eta)}{(2 u+\eta)} K^{(2)}\left(u+\frac{1}{2} \eta\right) b_{0}(u) \frac{Q^{(1)}(u-\eta) Q^{(2)}\left(u+\frac{3}{2} \eta\right)}{Q^{(1)}(u) Q^{(2)}\left(u+\frac{1}{2} \eta\right)} \\
& -K^{(3)}\left(u+\frac{1}{2} \eta\right) b_{0}(u) \frac{Q^{(2)}\left(u-\frac{1}{2} \eta\right)}{Q^{(2)}\left(u+\frac{1}{2} \eta\right)}-2 u(2 u-\eta) b_{0}(u) \frac{h Q^{(1)}(u-\eta)}{Q^{(2)}\left(u+\frac{1}{2} \eta\right)}
\end{aligned}
$$

where the $2 M$ Bethe roots must satisfy the BAEs (3.17) and (4.24), namely,

$$
\begin{aligned}
1 & +\frac{\left(2 \nu_{l}-\eta\right)}{\left(2 \nu_{l}+\eta\right)} \frac{K^{(2)}\left(\nu_{l}+\frac{1}{2} \eta\right)}{K^{(3)}\left(\nu_{l}+\frac{1}{2} \eta\right)} \frac{Q^{(1)}\left(\nu_{l}-\eta\right) Q^{(2)}\left(\nu_{l}+\frac{3}{2} \eta\right)}{Q^{(1)}\left(\nu_{l}\right) Q^{(2)}\left(\nu_{l}-\frac{1}{2} \eta\right)} \\
& =-h\left(2 \nu_{l}\right)\left(2 \nu_{l}-\eta\right) \frac{Q^{(1)}\left(\nu_{l}-\eta\right)}{K^{(3)}\left(\nu_{l}+\frac{1}{2} \eta\right) Q^{(2)}\left(\nu_{l}-\frac{1}{2} \eta\right)}, \quad l=1 \ldots M \\
1 & =\left(2 u_{k}-\eta\right) \frac{K^{(2)}\left(u_{k}+\frac{1}{2} \eta\right) b_{0}\left(u_{k}\right)}{K^{(1)}\left(u_{k}\right) a_{0}\left(u_{k}\right)} \frac{Q^{(2)}\left(u_{k}+\frac{3}{2} \eta\right)}{Q^{(2)}\left(u_{k}+\frac{1}{2} \eta\right)}, \quad k=1 \ldots M .
\end{aligned}
$$

In the homogeneous limit, the corresponding $T-Q$ relation and associated BAEs become (5.1) and (5.2)-(5.3) by setting $\theta_{j}=0, j=1, \ldots, N$. Therefore the energy of the Hamiltonian (1.1) reads

$$
\begin{aligned}
E & =-\left.\frac{\eta}{2} \frac{\partial \ln \Lambda(u)}{\partial u}\right|_{u=0,\left\{\theta_{j}\right\}=0}+\frac{\eta(2 c-1)}{2 \zeta}+\frac{\zeta^{\prime}}{\left(c^{\prime}-1 / 2\right) \eta-\zeta^{\prime}}-\mu M+L-1 \\
& =-\sum_{k=1}^{M} \frac{\eta^{2}}{u_{k}\left(u_{k}+\eta\right)}-\mu M,
\end{aligned}
$$

where the $2 M$ parameters $\left\{u_{j} \mid j=1, \ldots, M\right\}$ and $\left\{v_{j} \mid j=1, \ldots, M\right\}$ satisfy the resulting BAEs (5.2) and (5.3). Here we present the results for the $L=2$ and $L=3$ cases: the numerical solutions of the BAEs are shown in table 1 and table 2, which indicated that the eigenvalues are identical with the results we get from the exact diagonalization of the Hamiltonian (1.1). Numerical results for the small-site cases suggest that the spectrum obtained by the nested BAEs (5.2)-(5.3) is complete.

\footnotetext{
${ }^{2}$ Although the inhomogeneous $T-Q$ relation given by (5.1) is different from that obtained in [28], each of them gives rise to the complete set of eigenvalues of the transfer matrix. The $T-Q$ relation (5.1) takes advantage over one in [28] is that it leads to an simple form (4.27) of Bethe states of the reduced spectrum problem (3.16).
} 


\begin{tabular}{|cccc|c|c|}
\hline$u_{1}$ & $u_{2}$ & $\nu_{1}$ & $\nu_{2}$ & $E_{n}$ & $n$ \\
\hline$-0.1000-1.6602 i$ & $0.1004-0.0000 i$ & $0.1264+3.3108 i$ & $0.1264-3.3108 i$ & -5.312156 & 1 \\
$-0.1000-0.2048 i$ & $0.1004+0.0000 i$ & $0.8095-3.4060 i$ & $0.8095+3.4060 i$ & -4.555656 & 2 \\
$0.1005+0.0000 i$ & - & $0.0000-3.3070 i$ & - & -3.325040 & 3 \\
$-0.1000-1.6539 i$ & $-0.1000-0.2053 i$ & $0.0255+3.3085 i$ & $0.0255-3.3085 i$ & -3.218186 & 4 \\
$-0.1000-3.7095 i$ & $-0.1000-0.1000 i$ & $0.0000-4.6812 i$ & $0.1496-0.0000 i$ & -1.996355 & 5 \\
$-0.1000-2.3555 i$ & - & $0.0000-3.7060 i$ & - & -1.992804 & 6 \\
$-0.1000+0.2040 i$ & - & $-0.0000+3.3148 i$ & - & -1.225154 & 7 \\
- & - & - & - & 0 & 8 \\
$-0.1000-0.0999 i$ & - & $-0.1496-0.0000 i$ & - & 0.001822 & 9 \\
\hline
\end{tabular}

Table 1. Solutions of BAEs (5.2) and (5.3) where $L=2$ with the parameters $\eta=0.2, \mu=2$, $\zeta=0.1, c=0.1, c_{1}=-0.5, \zeta^{\prime}=-0.5, c^{\prime}=-0.3$ and $c_{1}^{\prime}=-0.7$ for the case of $E_{n}$ is the corresponding eigenenergy. The energy $E_{n}$ calculated from (5.4) is the same as that from the exact diagonalization of the Hamiltonian (1.1).

\section{Concluding remarks}

In this paper, we have studied the one-dimensional supersymmetric $t-J$ model with the most generic integrable boundary condition, which is described by the Hamiltonian (1.1) and the corresponding integrable boundary terms are associated with the most generic non-diagonal $K$-matrices given by (2.14)-(2.15). By combining the algebraic Bethe ansatz and the off-diagonal Bethe ansatz, we construct the eigenstates of the transfer matrix in terms of the nested Bethe states given by (3.6) and (4.27), which have well-defined homogeneous limit. The corresponding eigenvalues are given in terms of the inhomogeneous $T-Q$ relation (5.1) and the associated BAEs (5.2)-(5.3). The exact solution of this paper provides basis for further analyzing the thermodynamic properties and correlation functions of the model. These are under investigation and results will be reported elsewhere.

\section{Acknowledgments}

We would like to thank Prof. Y. Wang for his valuable discussions and continuous encouragements. The financial supports from the National Program for Basic Research of MOST (Grant No. 2016YFA0300600 and 2016YFA0302104), the National Natural Science Foundation of China (Grant Nos. 11434013, 11425522 and 11547045), the Major Basic Research Program of Natural Science of Shaanxi Province (Grant No. 2017ZDJC-32), BCMIIS and the Strategic Priority Research Program of the Chinese Academy of Sciences are gratefully acknowledged. 


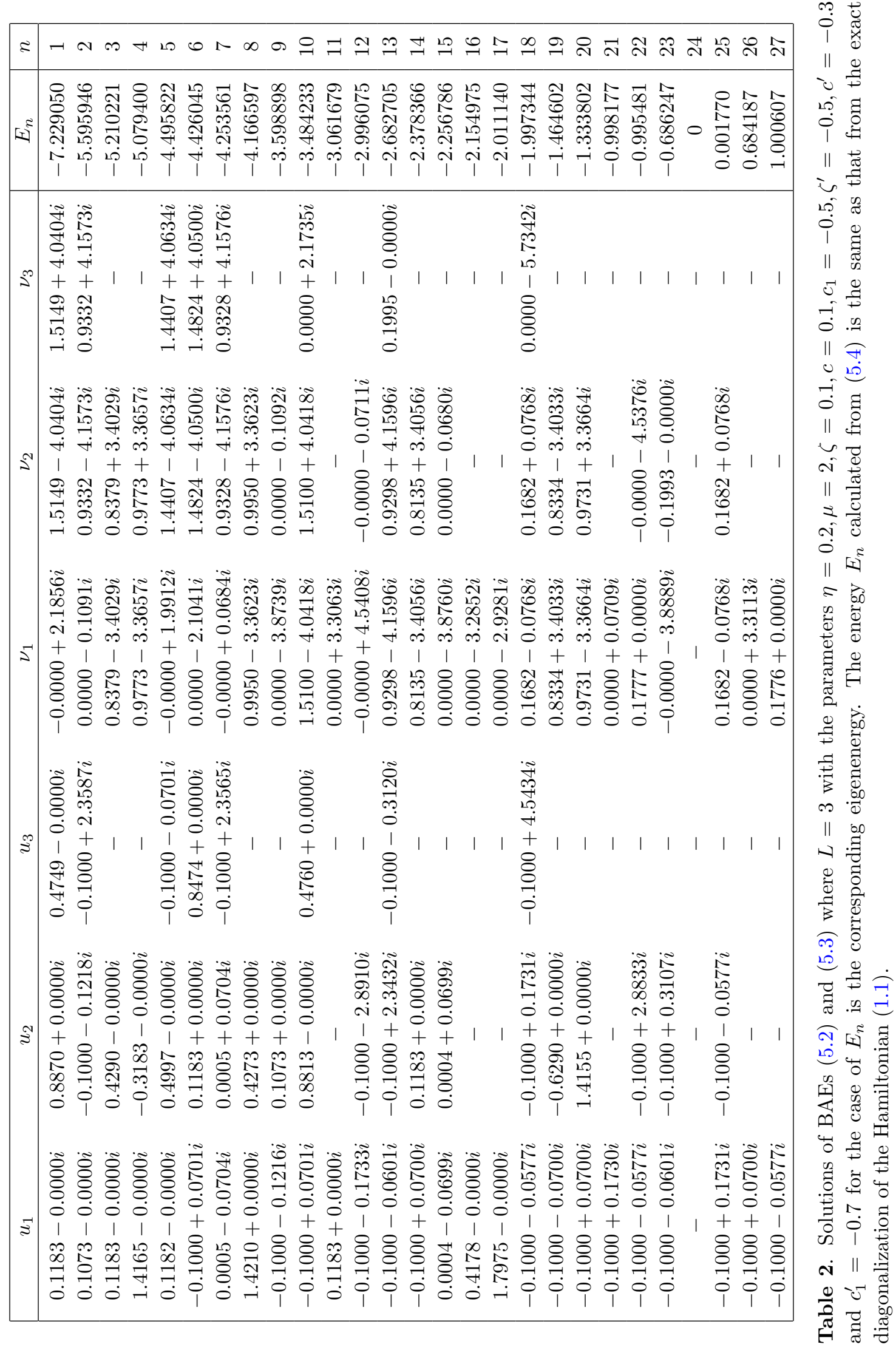


Open Access. This article is distributed under the terms of the Creative Commons Attribution License (CC-BY 4.0), which permits any use, distribution and reproduction in any medium, provided the original author(s) and source are credited.

\section{References}

[1] F.C. Zhang and T.M. Rice, Effective Hamiltonian for the superconducting Cüoxides, Phys. Rev. B 37 (1988) 3759.

[2] Z.-N. Hu and F.-C. Pu, Two magnetic impurities with arbitrary spins in open boundary $t-J$ model, Nucl. Phys. B 546 (1999) 691 [InSPIRE].

[3] H. Eskes and G.A. Sawatzky, Tendency towards local spin compensation of holes in the high-T $T_{c}$ copper compounds, Phys. Rev. Lett. 61 (1988) 1415.

[4] M.S. Hybertsen, M.S. Schlüter and N.E. Christensen, Calculation of Coulomb-interaction parameters for $\mathrm{La}_{2} \mathrm{CuO}_{4}$ using a constrained-density-functional approach, Phys. Rev. B 39 (1989) 9028.

[5] M.S. Hybertsen, E.B. Stechel, M. Schlüter and D.R. Jennison, Renormalization from density-functional theory to strong-coupling models for electronic states in $\mathrm{Cu}$-O materials, Phys. Rev. B 41 (1990) 11068.

[6] S. Reja, J.V.D. Brink and S. Nishimoto, Strongly Enhanced Superconductivity in Coupled $t-J$ Segments, Phys. Rev. Lett. 116 (2016) 067002 [arXiv:1509.04117].

[7] C.K. Lai, Lattice gas with nearest neighbor interaction in one dimension with arbitrary statistics, J. Math. Phys. 15 (1974) 1675.

[8] B. Sutherland, Model for a multicomponent quantum system, Phys. Rev. B 12 (1975) 3795.

[9] S. SARKAR, The supersymmetric $t-J$ model in one dimension, J. Phys. A 24 (1991) 1137 [INSPIRE].

[10] A. Foerster and M. Karowski, The Supersymmetric $t-J$ model with quantum group invariance, Nucl. Phys. B 408 (1993) 512 [INSPIRE].

[11] A. González-Ruiz, Integrable open boundary conditions for the supersymmetric $t-J$ model. The Quantum group invariant case, Nucl. Phys. B 424 (1994) 468 [hep-th/9401118] [INSPIRE].

[12] F.H. Essler, The supersymmetric $t-J$ model with a boundary, J. Phys. A 29 (1996) 6183.

[13] Y. Wang, J. Dai, Z. Hu and F.C. Pu, Exact Results for a Kondo Problem in a One-Dimensional t - J Model, Phys. Rev. Lett. 79 (1997) 1901.

[14] H. Fan, B.-y. Hou and K.-j. Shi, Algebraic Bethe ansatz for the supersymmetric $t-J$ model with reflecting boundary conditions, Nucl. Phys. B 541 (1999) 483 [InSPIRE].

[15] Y.K. Zhou and M.T. Batchelor, Spin excitations in the integrable open quantum group invariant supersymmetric $t-J$ model, Nucl. Phys. B 490 (1997) 576 [InSPIRE].

[16] H. Fan and M. Wadati, Integrable boundary impurities in the $t-J$ model with different gradings, Nucl. Phys. B 599 (2001) 561 [InSPIRE].

[17] H. Fan, M. Wadati and R.H. Yue, Boundary impurities in the generalized supersymmetric $t-J$ model, J. Phys. A 33 (2000) 6187. 
[18] G. Bedürftig and H. Frahm, Open $t-J$ chain with boundary impurities, J. Phys. A 32 (1999) 4585.

[19] Z.H. Hu, F.C. Pu and Y. Wang, Integrabilities of the $t-J$ model with impurities, J. Phys. A 31 (1998) 5241.

[20] W. Galleas, Spectrum of the supersymmetric $t-J$ model with non-diagonal open boundaries, Nucl. Phys. B 777 (2007) 352 [InSPIRE].

[21] J. Cao, W. Yang, K. Shi and Y. Wang, Off-diagonal Bethe ansatz and exact solution of a topological spin ring, Phys. Rev. Lett. 111 (2013) 137201 [arXiv:1305.7328] [INSPIRE].

[22] Y. Wang, W.L. Yang, J. Cao and K. Shi, Off-diagonal Bethe ansatz for exactly solvable models, Springer, Heidelberg, Germany (2015).

[23] J. Cao, W.-L. Yang, K. Shi and Y. Wang, Off-diagonal Bethe ansatz solutions of the anisotropic spin- $\frac{1}{2}$ chains with arbitrary boundary fields, Nucl. Phys. B 877 (2013) 152 [arXiv: 1307.2023] [INSPIRE].

[24] J. Cao, W.-L. Yang, S. Cui, K.-J. Shi and Y. Wang, Spin- $\frac{1}{2}$ XYZ model revisit: General solutions via off-diagonal Bethe ansatz, Nucl. Phys. B 886 (2014) 185 [arXiv:1307.0280] [INSPIRE].

[25] Y.-Y. Li, J. Cao, W.-L. Yang, K. Shi and Y. Wang, Exact solution of the one-dimensional Hubbard model with arbitrary boundary magnetic fields, Nucl. Phys. B 879 (2014) 98 [arXiv: 1311.0432] [INSPIRE].

[26] J. Cao, W.-L. Yang, K. Shi and Y. Wang, Nested off-diagonal Bethe ansatz and exact solutions of the $\mathrm{SU}(N)$ spin chain with generic integrable boundaries, JHEP 04 (2014) 143 [arXiv: 1312.4770] [INSPIRE].

[27] K. Hao, J. Cao, G.-L. Li, W.-L. Yang, K. Shi and Y. Wang, Exact solution of the Izergin-Korepin model with general non-diagonal boundary terms, JHEP 06 (2014) 128 [arXiv:1403.7915] [INSPIRE].

[28] X. Zhang, J. Cao, W.-L. Yang, K. Shi and Y. Wang, Exact solution of the one-dimensional super-symmetric $t-J$ model with unparallel boundary fields, J. Stat. Mech. 1404 (2014) P04031 [arXiv:1312.0376] [INSPIRE].

[29] F.H. Essler and V.E. Korepin, Higher conservation laws and algebraic Bethe Ansatze for the supersymmetric $t-J$ model, Phys. Rev. B 46 (1992) 9147.

[30] A.M. Grabinski and H. Frahm, Truncation identities for the small polaron fusion hierarchy, New J. Phys. 15 (2013) 043026 [arXiv:1211.6328] [InSPIRE].

[31] H. Fan, M. Wadati and X.M. Wang, Exact diagonalization of the generalized supersymmetric $t-J$ model with boundaries, Phys. Rev. B 61 (2000) 3450.

[32] A.J. Bracken, X.-Y. Ge, Y.-Z. Zhang and H.-Q. Zhou, Integrable open-boundary conditions for the q-deformed supersymmetric $U$ model of strongly correlated electrons, Nucl. Phys. B 516 (1998) 588 [INSPIRE].

[33] M.D. Gould, Y.Z. Zhang and H.Q. Zhou, Eight-state supersymmetric U model of strongly correlated fermions, Phys. Rev. B 57 (1998) 9498 [cond-mat/9709129].

[34] J. Cao, W.-L. Yang, K. Shi and Y. Wang, Off-diagonal Bethe ansatz solution of the XXX spin-chain with arbitrary boundary conditions, Nucl. Phys. B 875 (2013) 152 [arXiv: 1306.1742] [INSPIRE]. 
[35] R.I. Nepomechie, An inhomogeneous $T-Q$ equation for the open $X X X$ chain with general boundary terms: completeness and arbitrary spin, J. Phys. A 46 (2013) 442002 [arXiv: 1307.5049] [INSPIRE].

[36] S. Belliard and N. Crampé, Heisenberg XXX Model with General Boundaries: Eigenvectors from Algebraic Bethe Ansatz, SIGMA 9 (2013) 072 [arXiv:1309.6165] [INSPIRE].

[37] X. Zhang, Y.-Y. Li, J. Cao, W.-L. Yang, K. Shi and Y. Wang, Retrieve the Bethe states of quantum integrable models solved via off-diagonal Bethe Ansatz, J. Stat. Mech. 1505 (2015) P05014 [arXiv: 1407.5294] [INSPIRE].

[38] H. Frahm, A. Seel and T. Wirth, Separation of Variables in the open XXX chain, Nucl. Phys. B 802 (2008) 351 [arXiv: 0803.1776] [InSPIRE]. 